\title{
Superconductor Requirements and Characterization for High Field Accelerator Magnets
}

\author{
E. Barzi, A. V. Zlobin \\ Fermi National Accelerator Laboratory (FNAL) \\ Pine and Kirk Rds, Batavia, IL 60510, U.S. \\ E-mail: barzi@fnal.gov
}

\begin{abstract}
The 2014 Particle Physics Project Prioritization Panel (P5) strategic plan for U.S. High Energy Physics (HEP) endorses a continued world leadership role in superconducting magnet technology for future Energy Frontier Programs. This includes 10 to $15 \mathrm{~T} \mathrm{Nb}_{3} \mathrm{Sn}$ accelerator magnets for LHC upgrades and a future $100 \mathrm{TeV}$ scale $p p$ collider, and as ultimate goal that of developing magnet technologies above $20 \mathrm{~T}$ based on both High Temperature Superconductors (HTS) and Low Temperature Superconductors (LTS) for accelerator magnets. To achieve these objectives, a sound conductor development and characterization program is needed and is herein described. This program is intended to be conducted in close collaboration with U.S. and International labs, Universities and Industry.
\end{abstract}

Keywords—accelerator magnet; HTS; LTS; Rutherford cable

\section{INTRODUCTION}

A dual goal for superconducting (SC) magnets within the U.S. General Accelerator R\&D (GARD) is that of increasing performance and decreasing costs to enable the technology for a $100 \mathrm{TeV}$ scale proton-proton collider [1], [2]. This would be best achieved by exploiting the decade-long investment in the $\mathrm{Nb}_{3} \mathrm{Sn}$ technology, which produced at FNAL the first series of 10 to $12 \mathrm{~T}$ accelerator-quality dipoles and quadrupoles, as well as their scale-up [3]-[5]. Such advanced $\mathrm{Nb}_{3} \mathrm{Sn}$ technology can now be pushed up to its limits of $\sim 15 \mathrm{~T}$ field by improving $\mathrm{Nb}_{3} \mathrm{Sn}$ strands and cables, and developing and implementing further innovative approaches. The ultimate goal of the GARD SC magnet program is that of developing HTS inserts producing fields larger than $5 \mathrm{~T}$ within $15 \mathrm{~T} \mathrm{Nb}_{3} \mathrm{Sn}$ outserts to generate $20 \mathrm{~T}$ or higher fields for future high energy colliders.

In a sound magnet $R \& D$ program, the set of functional specifications (i.e. magnet aperture and length, operating field range and field quality, alignment tolerances, cryogenic and power requirements, magnet quench protection, etc.) for an accelerator magnet are prepared in tight conjunction with accelerator experts. Conceptual design studies of magnets that meet the agreed specifications are then performed. This step goes hand in hand with studies and optimization of the superconductor and cable properties, and evolves and changes along with superconductor progress. For this reason, it is vital to pursue an assertive conductor $\mathrm{R} \& \mathrm{D}$ program in parallel to magnet development.

Work supported by Fermi Research Alliance, LLC, under contract No. DEAC02-07CH11359 with the U.S. Department of Energy.
The robust and versatile infrastructure that was developed at FNAL in support of advanced superconductor and accelerator magnet development, together with the expertise acquired by the magnet scientists and engineers in design and analysis tools for SC materials, cable and coil technologies, makes FNAL an ideal setting for exploring SC accelerator magnets at the limit of $\mathrm{Nb}_{3} \mathrm{Sn}$ capabilities and beyond. Also, since 1998 the FNAL SC R\&D Lab has served as ideal stage for hands-on training of 34 graduate students in Physics and Engineering on superconductivity, mechanics, heat transfer, cryogenics, electronics, computing and automation during summer internships or Specialized Laurea or $\mathrm{PhD}$ theses.

This paper identifies and details key research activities within the superconductor $R \& D$ area that are required to help achieve the magnet program goals, and describe methods used at FNAL to address these topics.

\section{MAGNET AND CONDUCTOR R\&D STRATEGY}

The mission of the SC R\&D program is to understand and improve scientific and engineering aspects of SC strands and cables that are used in accelerator magnets, including LTS magnets and HTS inserts. The outcome of this effort provides conductor specifications and essential engineering data for design and construction of SC accelerator magnets. Coordination with industry has been critical to improve the SC performance of strands in cables, and collaboration with other laboratories and universities has contributed to their fundamental understanding at all levels.

By $2015, \mathrm{Nb}_{3} \mathrm{Sn}$ will have completed its maturity as an engineering material to be used in $11 \mathrm{~T}$ dipoles and interaction region (IR) quadrupoles for the LHC upgrades [6]. Magnets at the $15 \mathrm{~T}$ limit of the $\mathrm{Nb}_{3} \mathrm{Sn}$ technology will require continued development and optimization of $\mathrm{Nb}_{3} \mathrm{Sn}$ strands and cables, focusing on the performance at high fields.

Magnets at or above $20 \mathrm{~T}$ for future colliders will require materials outside of the Niobium family. Conductor, cable and insert coil development must therefore be emphasized in strategic plans. These include Bi-2212 round wires and REBCO tapes, as well as the most appropriate cables and ancillary materials required for insulation, etc. This work is integrated with that at other labs funded by DOE to make highcurrent cables, coils inserts, investigate their properties, and 
apply the fundamental work topics developed for $\mathrm{Nb}_{3} \mathrm{Sn}$ superconductors to HTS materials.

Consistently with the integrated strategy proposed for a U.S. National program [7], the primary thrust for the first couple of years of the SC Magnet program at FNAL will push for $15 \mathrm{~T} \mathrm{Nb}_{3} \mathrm{Sn}$ accelerator magnets to serve as a baseline platform for future circular colliders. For maximum costeffectiveness, wherever possible the program will use existing infrastructure and proven tools instead of ex novo equipment design. The existing $11 \mathrm{~T}$ dipole magnet developed for the LHC collimation system upgrade will be extended to achieve $15 \mathrm{~T}$ field in $60 \mathrm{~mm}$ aperture by the addition of two coil layers. This approach will use existing tooling, components and techniques developed at FNAL for the $11 \mathrm{~T}$ dipole program and LARP HQ quadrupole. This magnet will be available as a test platform for the first HTS insert coils developed in the U.S. and elsewhere. In parallel, design studies for cost optimized twin aperture $15 \mathrm{~T}$ dipoles will be performed and available for the conceptual design report on Future Circular Colliders (FCC) to be released at CERN in 2018.

In a mid-term phase, as an important step towards a $20 \mathrm{~T}$ dipole, a large aperture $15 \quad \mathrm{~T} \quad \mathrm{Nb}_{3} \mathrm{Sn}$ dipole with stress management solutions will be developed and tested. The large aperture $\mathrm{Nb}_{3} \mathrm{Sn}$ dipole will provide a test bed to accommodate an evolving Bi-2212 or REBCO technology as inserts. This activity plays a key role and has the potential for very high impact on the future Energy Frontier programs in the U.S. and worldwide, including energy upgrades for the LHC as well as large aperture, high field magnets for the FCC and for a Muon Collider Storage Ring.

\section{CONDUCTOR REQUIREMENTS}

A most pressing goal for strand development in the SC Materials program is to reach production level for the best possible conductor, which meets as closely as possible the desired target requirements. These include optimization of the critical current density $J_{c}$ at the operation field and temperature, balancing strand size and effective filament diameter $d_{\text {eff }}$ for thermo-magnetic stability and low magnetization, and assessing $\mathrm{Cu}$ to non- $\mathrm{Cu}$ ratio and Residual Resistivity Ratio $(R R R)$ for a reliable quench protection. In addition, the effects of the cabling process on these same parameters have to be known, as well as any $J_{c}$ reduction and/or irreversible degradation due to magnet assembly, cool-down and operation.

Appealing materials for use in high-field accelerator magnets include $\mathrm{Nb}_{3} \mathrm{Sn}, \mathrm{Nb}_{3} \mathrm{Al}$ and $\mathrm{Bi}-2212$. All of these are produced in the form of composite round wires. Cross-sections of these wires are shown in Fig. 1. Their actual potential for high-field accelerator magnets is being extensively studied.

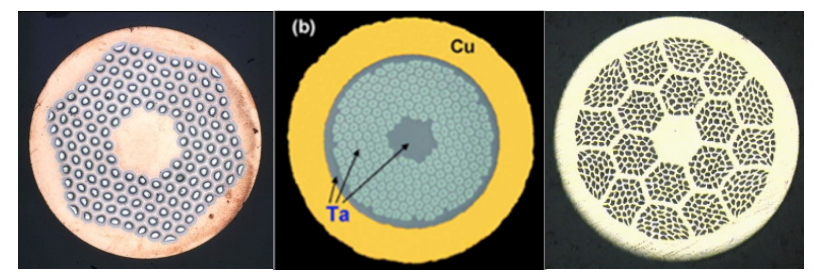

Fig. 1. $\mathrm{Nb}_{3} \mathrm{Sn}$ (left), $\mathrm{Nb}_{3} \mathrm{Al}$ (center) and $\mathrm{Bi}-2212$ (right) composite wires. Courtesy of OST and NIMS.
$\mathrm{Nb}_{3} \mathrm{Sn}$ is an intermetallic compound, the second $\mathrm{SC}$ material most widely used in high-field magnet applications. FNAL has focused on developing the Restacked-Rod-Process (RRP®) technology with Oxford Instruments Superconducting Technology (OST), producing billets of 102, 108 and 114 subelements over 127 restacks and of 132 and 150 subelements over 169 restacks, using both Ta and Ti as ternary element in the $\mathrm{Nb}_{3} \mathrm{Sn}$. These designs meet the specs in the form of 0.7 to $1 \mathrm{~mm}$ round wire and also withstand the plastic deformation occurring in Rutherford cabling. In the meantime, the U.S. DOE Conductor Development Program (CDP), after a successful effort to improve the conductor $J_{c}(12 \mathrm{~T}, 4.2 \mathrm{~K})$ from 1500 to $>3000 \mathrm{~A} / \mathrm{mm}^{2}$, has been aiming at further increasing the number of stacks in RRP® wires by sponsoring fabrication of 217 subelement billets at OST.

For cost-effective $15 \mathrm{~T}$ accelerator magnet designs, the $\mathrm{Nb}_{3} \mathrm{Sn}$ critical current density $J_{c}(12 \mathrm{~T}, 4.2 \mathrm{~K})$ will have to be pushed to $3500 \mathrm{~A} / \mathrm{mm}^{2}$, with $J_{c}(15 \mathrm{~T}, 4.2 \mathrm{~K}) \sim 2000 \mathrm{~A} / \mathrm{mm}^{2}$. In addition, whereas a large number of existing $\mathrm{Nb}_{3} \mathrm{Sn}$ magnet models have been made with relatively small strand sizes (i.e. 0.7 to $1 \mathrm{~mm}$ ), $15 \mathrm{~T}$ magnets will require much wider cables to lower the cable aspect ratio, reduce the number of coil layers, and decrease the number of turns, thereby the inductance which, in turn, simplifies magnet quench protection and allows increasing the magnet length. It is therefore crucial to drive $\mathrm{Nb}_{3} \mathrm{Sn}$ strand development further, and produce larger wires with adequate number of stacks to meet field quality requirements. Analysis and coil magnetic measurements show that $\mathrm{Nb}_{3} \mathrm{Sn}$ wires with subelement sizes of $\sim 40 \mu \mathrm{m}$ provide an acceptable level of coil magnetization effects.

$\mathrm{Nb}_{3} \mathrm{Al}$ composite wires have lower strain sensitivity than $\mathrm{Nb}_{3} \mathrm{Sn}$ and thus, real potential for operation at higher Lorentz forces. The superconducting $\mathrm{Nb}_{3} \mathrm{Al}$ phase is formed by the Rapid-Heating Quenching Transformation (RHQT) process developed by the National Institute for Materials Science (NIMS) in Japan. The $\mathrm{Cu}$ stabilizer is added by ion-plating followed by electro-plating after the RHQT process. The piece length of the wire has presently reached a few kilometers, but there are still significant fabrication challenges to be solved to further improve the $J_{c}$ and make $\mathrm{Nb}_{3} \mathrm{Al}$ cost and $J_{c}$ competitive with $\mathrm{Nb}_{3} \mathrm{Sn}$. In collaboration with NIMS and KEK, FNAL studies cabling capabilities of state-of-the-art $\mathrm{Nb}_{3} \mathrm{Al}$ strands.

Significant progress was made in the development and industrialization of $\mathrm{Bi}-2212$. Km-length quantities of $\mathrm{Bi}-2212$ composite round wires were commercially produced in the past decade. The $J_{c}(20 \mathrm{~T}, 4.2 \mathrm{~K})$ of the round wire is at or below $1500 \mathrm{~A} / \mathrm{mm}^{2}$, with an engineering current density $J_{e}(20 \mathrm{~T}$, $4.2 \mathrm{~K}$ ) over the whole wire cross-section of $400 \mathrm{~A} / \mathrm{mm}^{2}$ or less when heat treated at atmospheric pressure. $\mathrm{Bi}-2212$ requires a multistage heat treatment in highly corrosive Oxygen atmosphere at very uniform temperatures up to $900^{\circ} \mathrm{C}$. It was shown that increasing the $\mathrm{O}_{2}$ pressure during heat treatment up to $\sim 100$ bar provides a substantial improvement of the $J_{c}$, but this still has to be demonstrated on cables and coils. Also, this process does not seem to affect the high sensitivity of Bi-2212 to strain [8], which limits applications in high-field magnets, whose cables are typically subject to large transverse and longitudinal strains or stresses. In collaboration with U.S. labs and OST, FNAL studies Bi-2212 strand $I_{c}$ degradation due to plastic deformation in cables and due to axial and transverse pressure on the superconductor during magnet operation. 
The large aperture $15 \mathrm{~T}$ dipole magnets that are planned by 2020 will produce stresses in the coil that are known to exceed the present $J_{c}$ irreversibility limits. A solution will be to develop and implement innovative stress management solutions. At the same time, the ability of mapping and monitoring the effects of the 3D coil stresses on the conductor performance itself is a goal that has not yet been achieved and should ideally be included in a conductor plan.

Composite conductors with much higher $J_{c}$ would solve a majority of the existing challenges in high-field accelerator magnets. It might be therefore worth investing in improving the inherent flux pinning mechanisms of SC materials.

Examples of traditional Rutherford cable based on $\mathrm{Nb}_{3} \mathrm{Sn}$, $\mathrm{Nb}_{3} \mathrm{Al}$ and Bi-2212 strands are shown in Fig. 2. As an alternative to using larger wires, composite and multi-stage cables are also being designed and studied. Cable development is performed by designing and fabricating samples of different geometries using state-of-the-art wires, with the purpose of studying the effect of cable parameters and processing on its performance. This includes for instance the sensitivity of electrical properties $(J c, R R R)$ and internal structure (diffusion barrier, subelement shape and spacing) to cable compaction, measurements of cable stability and AC losses, measurements of 3D cable expansion during the fabrication process and during reaction, effects of intermediate annealing when using a 2-pass fabrication process, etc. Rutherford cable finite element models that allows evaluating for each considered cable geometry what is the plastic strain seen by the strands during fabrication, what are the most critical strand locations, and predicting local damage whenever the failure mechanisms of a specific strand technology are known, were produced at FNAL to aid in cable design [9].
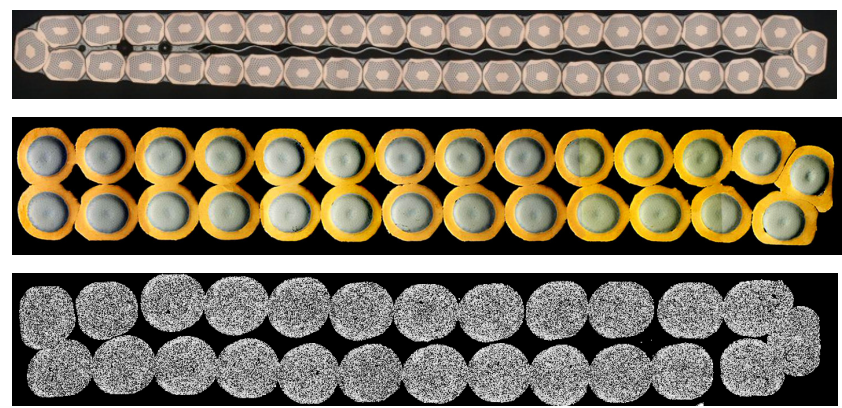

Fig. 2. Rutherford cable cross sections of: a) keystoned 40-strand $\mathrm{Nb}_{3} \mathrm{Sn}$ cable with $0.7 \mathrm{~mm}$ RRP150/169 strands and a $11 \mathrm{~mm}$ wide and $25 \mu \mathrm{m}$ thick stainless steel core (top) [9]; b) 27 -strand $(1 \mathrm{~mm}) \mathrm{Nb}_{3} \mathrm{Al}$ rectangular cable (middle) [10]; c) 25-strand (0.8 mm) Bi-2212 rectangular cable (bottom) [11].

\section{EXPERIMENTAL CAPABILITIES}

The infrastructure and experimental equipment of the SC R\&D Lab at FNAL was described in detail in [9]. Herein the types of measurements that can be performed are listed. The following measurements are performed in magnetic cryostats equipped with Variable Temperature Inserts (VTI) of $49 \mathrm{~mm}$ inner diameter:

- Critical current of round wires, flat-rolled wires, and strands extracted from cables up to $15 \mathrm{~T}, 2 \mathrm{kA}$ at $1.9-100 \mathrm{~K}$.
- Magnetization measurements between 0 and $15 \mathrm{~T}$ and at 1.9-100 K, and effective filament diameter $d_{\text {eff }}$, of SC wires and bulk samples using a balanced coil magnetometer.

- Residual Resistivity Ratio (RRR) of SC wires and bulk samples.

- Critical current sensitivity of SC wires to tensile and compressive strain up to $\sim 0.1 \%, 15 \mathrm{~T}, 2 \mathrm{kA}$ at $1.9-100 \mathrm{~K}$.

- Angular dependence of critical current of anisotropic HTS wires with respect to magnetic field direction up to $15 \mathrm{~T}$, $2 \mathrm{kA}$ at $1.9-100 \mathrm{~K}$.

- Electrical resistance of SC cable splices at self-field up to $28 \mathrm{kA}$ at $1.9-100 \mathrm{~K}$ using a SC transformer equipped with heater and Rogowski coil.

The following measurements are performed in the main $\mathrm{He}$ bath of cryostats equipped with $15 \mathrm{~T}$ and $14 \mathrm{~T}$ solenoids of respectively $64 \mathrm{~mm}$ and $77 \mathrm{~mm}$ inner diameters:

- Rutherford cable test at $4.2 \mathrm{~K}$ up to $28 \mathrm{kA}$ by means of a SC transformer with bifilar sample.

- Critical current sensitivity of Rutherford cable samples under uniaxial transverse load up to $200 \mathrm{MPa}$.

In some devices and model magnets, resistive strain gauges are used to measure stress and strain, and thermocouples are used to monitor the temperature in four tube furnaces for heat treatment of superconductors in Argon and Oxygen up to $1500^{\circ} \mathrm{C}$, and in a $2 \mathrm{~m}$ long furnace for heat treatment in air and in Argon up to $1250^{\circ} \mathrm{C}$. Strain gauge calibration is performed in a He temperature cell loader, and thermocouple calibration up to $700^{\circ} \mathrm{C}$ in a metrology well.

The Lab has also the following capabilities:

- 42-strand compact cabling machine to make Rutherford and composite round cables from 5 to $17.75 \mathrm{~mm}$ in width. Cables have been fabricated with and without stainless steel core, out of both conventional conductors like $\mathrm{Cu}$ and $\mathrm{Ag}$, and superconducting round wires, including $\mathrm{NbTi}_{1} \mathrm{Nb}_{3} \mathrm{Sn}$, $\mathrm{Nb}_{3} \mathrm{Al}, \mathrm{MgB}_{2}$ and $\mathrm{Bi}-2212$.

- A motorized flat-rolling system to impart plastic strain within a desired range to round SC wires, usually before heat treatment, for superconductor studies [9]. The strand is flattened vertically, and it is free to expand laterally.

- A Modular Insert Fixture (MIF) to test YBCO double pancake coils inside a $\mathrm{SC}$ solenoid. It can be used also to test insert coils wound with Bi-2212 strands or small cables.

- A 50 liters cryostat with $253 \mathrm{~mm}$ neck. The power supply provides $2400 \mathrm{~A}$. The DAQ hardware and software systems include current, voltages, temperature, magnetic field and helium level readings, and quench protection.

- A 75 liters magnetic cryostat with SC solenoid of $8.5 \mathrm{~T}$ at $4.2 \mathrm{~K}$ and $10 \mathrm{~T}$ at the Lambda point, with a cold aperture of $147 \mathrm{~mm}$. The power supply presently provides $2400 \mathrm{~A}$. The DAQ hardware and software systems include current, voltages, temperature, magnetic field and helium level readings, and quench protection. The cryostat can be used in the future to test HTS coils up to $146 \mathrm{~mm}$ in OD and/or cables.

\section{CONDUCTOR CHARACTERIZATION}

The following wire procurement and qualification plan, to evaluate and select the most adequate conductors, is the first 
important step towards the ultimate test of the conductor in magnet models:

- Procurement of billets from industry in sufficiently large quantities to verify production quality.

- Qualification of round and deformed strands for $J_{c}$, flux jump stability, $d_{e f f}, R R R$, and $J_{c}$ strain sensitivity.

- Rutherford cable development and fabrication.

- Characterization of strands extracted from cables.

- Cable test.

An example of the characterization steps from the above process are shown in the following.

A. Qualification of Round and Deformed Strands

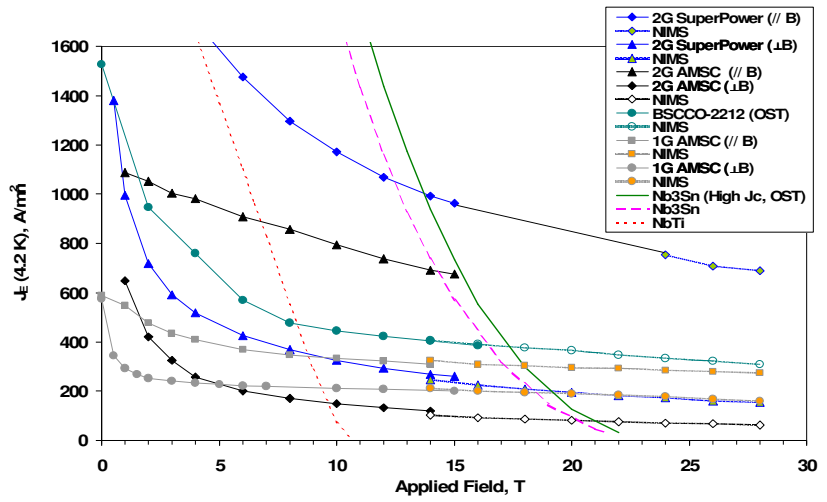

Fig. 3. Engineering critical current density at $4.2 \mathrm{~K}$ of a number of LTS and HTS conductors as a function of magnetic field [12].

1) $J_{c}$ of Round Wires: The $J_{c}$ of the round wire is a key starting point for magnet design. In standard strand $I_{c}$ measurements, three pairs of voltage taps are used. Two pairs are placed along the center of the spiral sample $50 \mathrm{~cm}$ and $75 \mathrm{~cm}$ apart, and one pair at the $\mathrm{Cu}$ leads to be used for quench protection. The $I_{c}$ is determined from the voltage-current $(V-I)$ curve using either the $10^{-14} \Omega \cdot \mathrm{m}$ resistivity or an electrical field criterion. Typical $I_{c}$ measurement uncertainties are within $\pm 1 \%$ at $4.2 \mathrm{~K}$ and $12 \mathrm{~T}$. Fig. 3 shows as an example the engineering critical current density at $4.2 \mathrm{~K}$ of a number of LTS and HTS conductors obtained as a function of magnetic field [12].

2) Flux Jump Stability: State-of-the-art high $J_{c} \quad \mathrm{Nb}_{3} \mathrm{Sn}$ strands are subject to flux jumps (Fig. 5) when their subelement sizes are larger than some threshold values. The data of $\mathrm{Nb}_{3} \mathrm{Sn}$ magnet models tested between 10 and $13 \mathrm{~T}$ has shown that for stable magnet performance down to $1.9 \mathrm{~K}$, subelement size has to be $\leq 40 \mu \mathrm{m}$ with conductor $J_{c}(12 \mathrm{~T}$, $4.2 \mathrm{~K}$ ) of $2500-3000 \mathrm{~A} / \mathrm{mm}^{2}$. Unstable magnet performance occurs when the load line of the magnet encompasses an instability region in the $I$ vs. $B$ conductor behavior. Wires are indicatively used to determine the maximum quench current in the presence of a magnetic field variation by performing $\mathrm{V}-\mathrm{H}$ tests in addition to standard $V$ - $I$ tests. In $V$ - $H$ tests the transport current is ramped to a fixed value, and the field is swept up and down between 0 and $4 \mathrm{~T}$ with ramp rates of 5 to $17 \mathrm{mT} / \mathrm{s}$. If no quench is observed the current is increased and the test repeated.

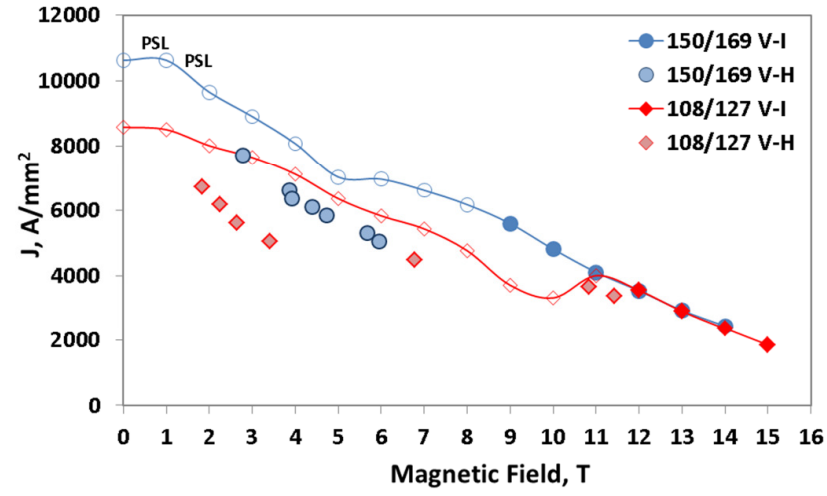

Fig. 4. $V-I$ and $V-H$ test results in superfluid $\mathrm{He}$ at $1.9 \mathrm{~K}$ of two different RRP® wires of same $J_{c}$ and size [13].

Fig. 4 shows as an example $V-I$ and $V$ - $H$ test results in superfluid $\mathrm{He}$ at $1.9 \mathrm{~K}$ of two RRP® wires with different subelement size [13]. Closed symbols represent $I_{c}$ values obtained from smooth $V-I$ transitions, whereas open symbols indicate the maximum quench currents $I_{q}$. In this example, the RRP108/127 wire had a geometrical subelement size 14\% larger than the RRP150/169 wire.

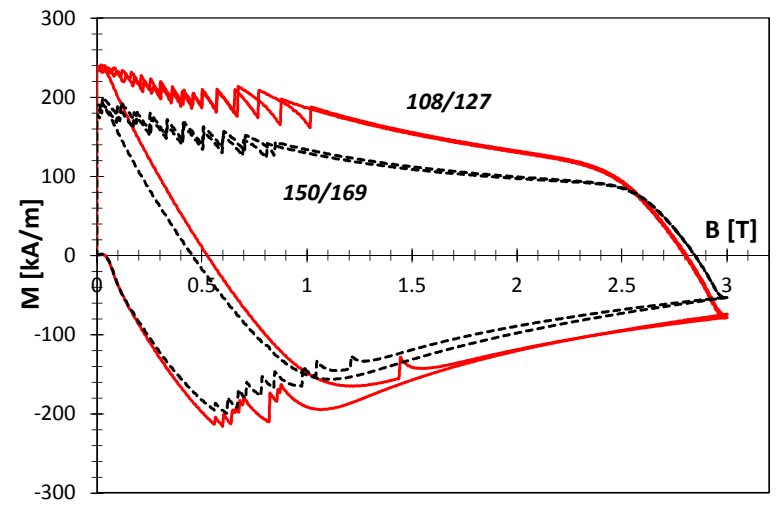

Fig. 5. Magnetization at $4.2 \mathrm{~K}$ per total strand volume for two $0.7 \mathrm{~mm} \mathrm{RRP} \circledR$ wires between 0 and $3 \mathrm{~T}$ [13].

3) Magnetization and $D_{\text {eff: }}$ An example of magnetization measurement performed at $4.2 \mathrm{~K}$ for two $0.7 \mathrm{~mm}$ RRP® wires between 0 and $3 \mathrm{~T}$ is plotted in Fig. 5 per total strand volume [13]. Both wires show flux jumps. The $d_{\text {eff }}$ can be obtained from the width of the magnetization loop $\Delta M(B) \propto J_{c}(B) \cdot d_{\text {eff }}$ using the measured $J_{c}(B)$ dependence.

4) $J_{c}$ of Deformed Wires: Superconducting wires are flatrolled to increasing deformations to systematically study such dependence of their properties. The $J_{c}$ retention at $4.2 \mathrm{~K}$ at a given field of $14 \mathrm{~T}$, expressed as the $I_{c}$ of a deformed strand normalized to the $I_{c}$ of the virgin wire, is shown in Fig. 6 as function of strand deformation for a number of RRP® wires [14]. Wire deformation is defined as $(d-t) / d$, where $d$ is the strand diameter and $t$ is the thickness of the deformed strand. These results are also used to anticipate how well a given billet withstands plastic deformation, which in Rutherford cables can reach maximumm values of 20 to $30 \%$. 


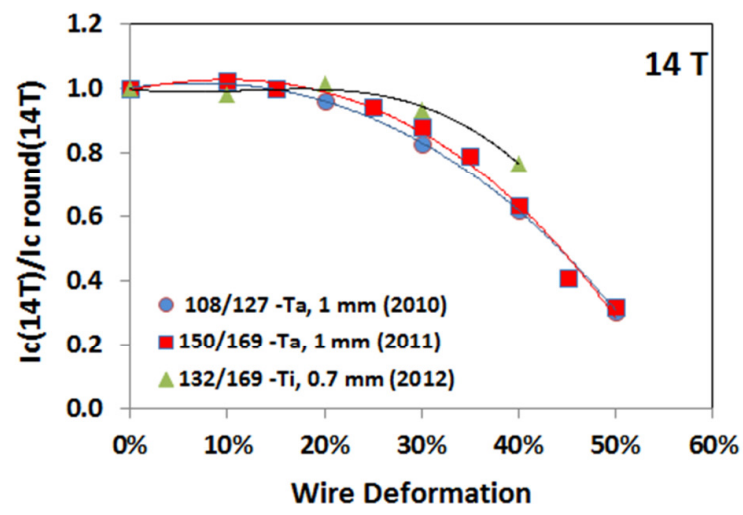

Fig. 6. $I_{c}(4.2 \mathrm{~K}, 14 \mathrm{~T})$ of rolled strand normalized to that of a round strand as a function of wire deformation for a number of RRP® wires [14].

5) RRR of Deformed Wires: The Residual Resistivity Ratio of a wire $\mathrm{Cu}$ matrix is a measure of its purity, which affects magnet stability and quench protection. Fig. 7 compares the $R R R$ of the rolled strand as a function of wire deformation for a number of $\mathrm{RRP} \circledast$ wires [14]. $\mathrm{Cu}$ purity depends substantially on billet composition and also on the heat teatment cycle used to form the $\mathrm{Nb}_{3} \mathrm{Sn}$ superconductor. In addition, $R R R$ is subject to a magneto-resistivity effect, by which $R R R$ strongly reduces at increasing magnetic fields.

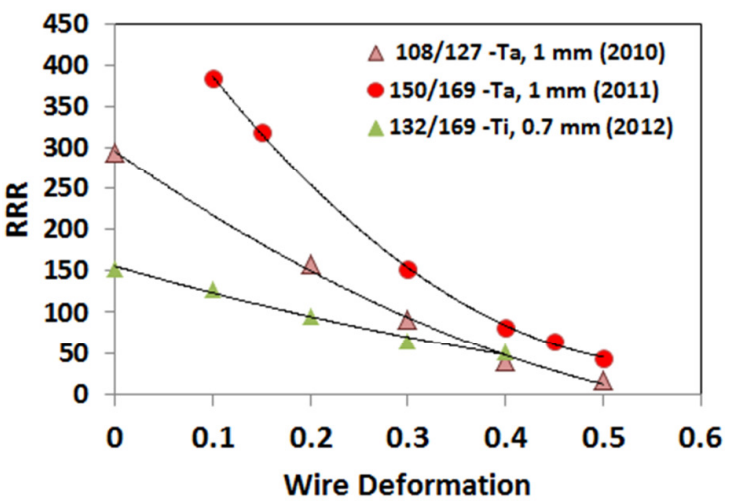

Fig. 7. RRR of the rolled strand at zero magnetic field as a function of wire deformation for a number of RRP® wires [14].

\section{B. $J_{c}$ Strain Sensitivity}

Since higher magnetic fields produce proportionally higher Lorentz forces, 3D strain sensitivity of critical current is a very important property in superconductors.

1) $J_{c}$ Sensitivity to Tensile/Compressive Strain: Measurements of $J_{c}$ strain sensitivity of SC wires are performed with a helical Walters' spring device, equipped with two concentric copper tubes used as $2 \mathrm{kA}$ current and $60 \mathrm{~N} \cdot \mathrm{m}$ torque carriers. The strain behavior for a number of superconductors is compared in Fig. 8, which shows the normalized $I_{c}(4.2 \mathrm{~K}, 15 \mathrm{~T})$ vs. applied strain. The irreversible strain can be also identified by going back to lower strain values. For instance, after reaching point $\mathrm{A}$ for one of the $\mathrm{Nb}_{3} \mathrm{Sn}$ wires, when going back to point $\mathrm{A}^{\prime}$ at lower applied strain, an $I_{c}$ value was obtained that was lower than the previous measurement by an amount larger than the $I_{c}$ measurement precision of $0.1 \mathrm{~A}$. This placed the irreversible intrinsic strain of the Ta-doped $\mathrm{Nb}_{3} \mathrm{Sn}$ wire at less than $+0.11 \%$, to be compared with the irreversible intrinsic strain range of $+0.26 \%$ to $+0.31 \%$ found for the Ti-doped wire, consistently also with NIST studies [15].

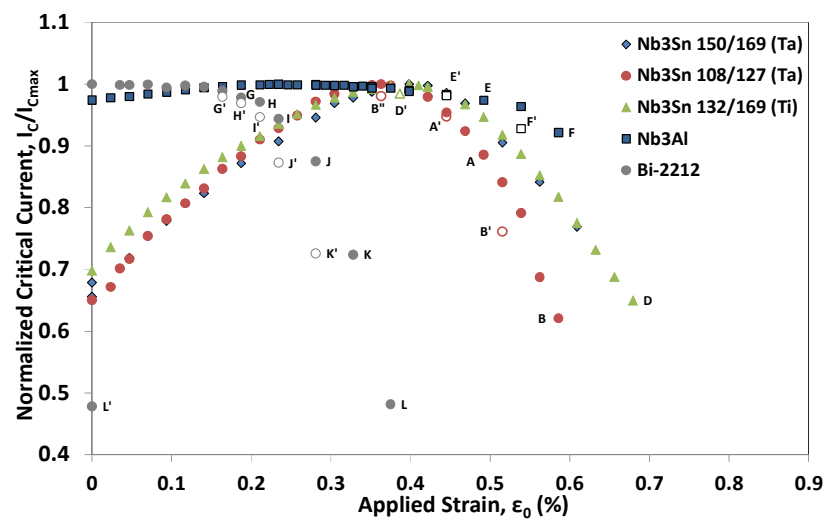

Fig. 8. Normalized $I_{c}(4.2 \mathrm{~K}, 15 \mathrm{~T})$ as function of longitudinal intrinsic strain for a number of $\mathrm{Nb}_{3} \mathrm{Sn} \mathrm{RRP} \circledast$ and $\mathrm{Nb}_{3} \mathrm{Al}$ wires.

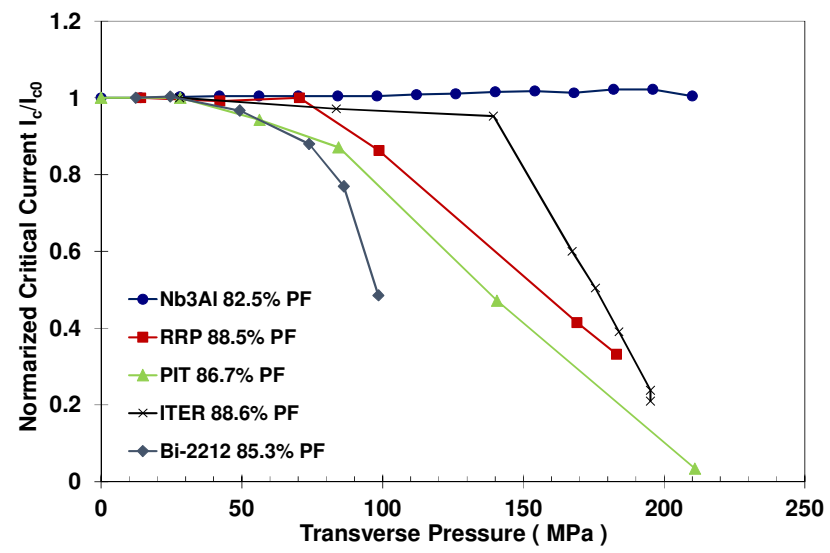

Fig. 9. Normalized $I_{c}(4.2 \mathrm{~K}, 12 \mathrm{~T})$ vs. uni-axial transverse pressure on Rutherford cable face for a number of $\mathrm{Nb}_{3} \mathrm{Sn}$ and $\mathrm{Nb}_{3} \mathrm{Al}$ conductors.

2) $J_{c}$ Sensitivity to Uni-axial Transverse Load: Fig. 9 shows the $I_{c}$ sensitivity of a number of superconductors to transverse pressures up to $210 \mathrm{MPa}$. It should be noted that these data represent the effect of uni-axial and not multi-axial strain, since the experimental setup allows for the sample to expand laterally. This produces larger strain values on the cable sample than for instance on a laterally contrained one, and therefore these are conservative results.

\section{Characterization of Strands Extracted from Cables}

Strands are extracted from cables before heat treatment and tested after reaction as a means to characterize a cable. During cable development, the $I_{c}$ at a given magnetic field and the $R R R$ of the extracted strand can be plotted against cable compaction and/or cable mid-thickness at each fabrication step. $I_{c}$ degradation values $<10 \%$ are typically desired. Assuming a uniform current distribution among the strands, cable $I_{c}$ is 
defined as the strand $I_{c}$ multiplied by the total number of strands in the cable. Increased flux jump instabilities in extracted strands indicate cable damage. Another important use of extracted strand tests is to include samples with the magnet coil during high temperature reaction as witnesses of the process.

\section{Cable Test}

1) Bi-2212 Rutherford Cable Test at Self-field: Bi-2212 cables can be tested at self-field with a SC transformer equipped with a Rogowski coil to measure the secondary current transported by the cable sample [11]. In Fig. 10 the cable critical surface obtained from extracted strand test results is compared with the transformer load line to produce the expected cable performance, or short sample limit predictions. This Bi-2212 Rutherford cable reached $90 \%$ of the current predicted by measuring extracted strands.

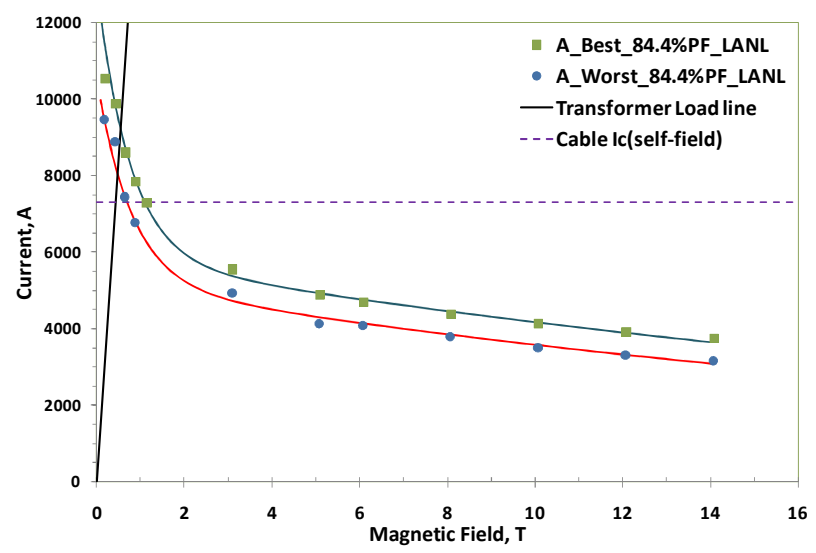

Fig. 10. Short sample limit predictions of Bi-2212 Rutherford cable $I_{c}$ based on extracted strand tests and the transformer load line [11].

2) $\mathrm{Nb}_{3} \mathrm{Sn}$ Rutherford Cable Test at Field: $I_{c}$ measurements of Rutherford cables are performed using a test fixture with bifilar sample and SC transformer that operates in the 14T/16T solenoid of a Teslatron system by Oxford Instruments. An example of cable quench current vs. magnetic field is shown in Fig. 11. The keystoned cable sample was made of 40 $0.7 \mathrm{~mm} \mathrm{RRP}{ }^{\circledR} \mathrm{Nb}_{3} \mathrm{Sn}$ strands and was heat treated together with witness samples of its extracted strands. Closed symbols represent the $I_{c}$ data in a $V-I$ transition, whereas open symbols denote $I_{q}$ in abrupt quenches. Self-field corrections were applied in this plot to both cable and strand test results [14]. The good correlation between strand and cable test results confirms uniform strand properties and uniform transport current distribution during the cable test.

\section{CONCLUSIONS}

Conductor properties determine key accelerator magnet parameters, including minimum and maximum operation fields, operation margins, field quality and quench protection. A preliminary list of superconductor specifications and a qualification plan was proposed and is being implemented at FNAL both on LTS and HTS conductors to evaluate the best candidates for high field accelerator magnets.

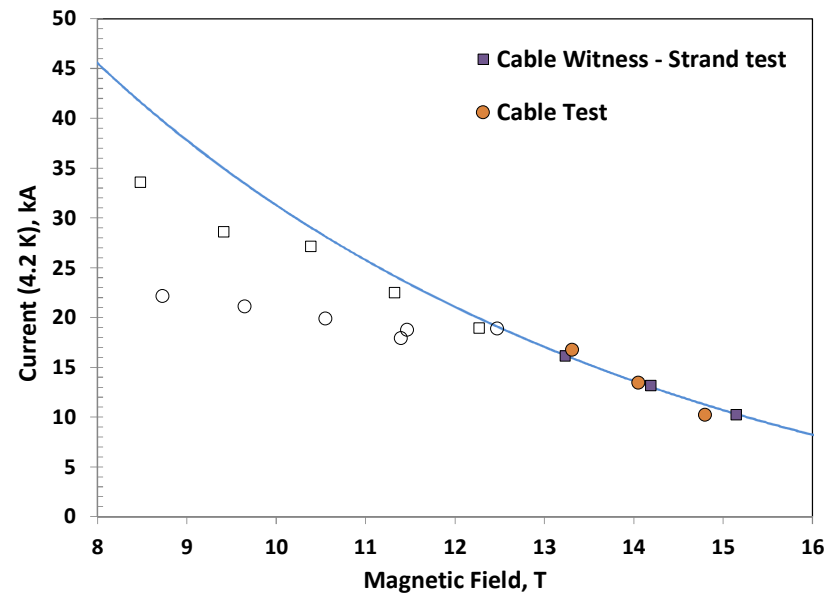

Fig. 11. Cable quench current vs. magnetic field for an insulated $\mathrm{Nb}_{3} \mathrm{Sn}$ cable sample made of $40 \mathrm{RRP} \circledast \mathrm{Nb}_{3} \mathrm{Sn}$ strands [14].

\section{ACKNOWLEDGMENT}

The authors thank their friend and colleague Dan Turrioni, as well as all the staff of the SC R\&D Lab.

\section{REFERENCES}

[1] "Building for Discovery: Strategic Plan for U.S. Particle Physics in the Global Context," P5 Report,

http://science.energy.gov/ /media/hep/hepap/pdf/May\%202014/FINAL _P5_Report_053014.pdf.

[2] Future Circular Collider Study Kickoff Meeting, Geneva, Feb. 12-14, 2014, http://indico.cern.ch/event/282344/timetable/\#20140212.

[3] A.V. Zlobin et al., "Development of $\mathrm{Nb}_{3} \mathrm{Sn}$ accelerator magnet technology at Fermilab", Proceedings of PAC2007, Albuquerque, NM, June 2007.

[4] R. Bossert et al., "Fabrication and Test of 4-m Long $\mathrm{Nb}_{3} \mathrm{Sn}$ Quadrupole Coil Made of RRP-114/127 Strand", Advances in Cryogenic Engineering, V. 57, AIP, V. 1434, pp. 869-876 (2012).

[5] A.V. Zlobin et al., "Status of the $11 \mathrm{~T}$ 2-in-1 $\mathrm{Nb}_{3} \mathrm{Sn}$ Dipole Development for LHC", Proceedings of IPAC2014, Dresden, June 2014.

[6] L. Bottura et al., "Advanced Accelerator Magnets for Upgrading the LHC", IEEE Trans. on Appl. Supercond., Vol. 22, Issue 3, 2012, p. 4002008.

[7] G. Apollinari et al., "National Program on High Field Accelerator Magnet R\&D,” FERMILAB-FN-0993-TD, September 26, 2014.

[8] A. Godeke, private communication.

[9] E. Barzi et al., "Superconducting Strand and Cable Development for the LHC Upgrades and Beyond," IEEE Trans. on Appl. Supercond., Vol. 23, Issue 3, June 2013 Page 6001112

[10] A. Kikuchi et al., "Cu Stabilized $\mathrm{Nb}_{3} \mathrm{Al}$ Strands for the High Field Accelerator Magnet”, IEEE Trans. on Appl. Sup., V. 18, p. 1026 (2008).

[11] E. Barzi, V. Lombardo, D. Turrioni, F. J. Baca, and T. G. Holesinger, "BSCCO-2212 Wire and Cable Studies", Trans. Appl. Sup., V. 21, No. 3, p. 2335 (2011).

[12] D. Turrioni et al., "Study of HTS Wires at High Magnetic Fields", IEEE Trans. on Appl. Sup., V. 19, No. 3, p. 3057 (2009).

[13] E. Barzi, M. Bossert, G. Gallo, V. Lombardo, D. Turrioni, R. Yamada, and A.V. Zlobin, "Studies of $\mathrm{Nb}_{3} \mathrm{Sn}$ Strands based on the Restacked-Rod Process for High-field Accelerator Magnets", IEEE Trans. Appl. Sup., V. 22, No. 3, pp. 6001405 (2012).

[14] E. Barzi, D. Turrioni and A. V. Zlobin, "Progress in $\mathrm{Nb}_{3} \mathrm{Sn}$ RRP Strand Studies and Rutherford Cable Development at FNAL", IEEE Trans. on Appl. Sup., 6000808, V. 24, No. 3 (2014).

[15] Cheggour N et al., Supercond. Sci. Technol. 23 (2010) 052002 